\title{
EQUAÇÕES DE PREDIÇÃo DOS VALORES ENERGÉTICOS DOS ALIMENTOS PARA PAPAGAIOS VERDADEIROS (Amazona aestiva)
}

\author{
Predition equation of energetic values of feeds for blue-fronted parrot \\ (Amazona aestiva)
}

\author{
Carlos Eduardo do Prado Saad ${ }^{1}$, Walter Motta Ferreira², Flávia Maria de Oliveira Borges ${ }^{3}$, \\ Leonardo Boscoli Lara ${ }^{4}$
}

\begin{abstract}
RESUMO
Objetivou-se com este trabalho estimar, a partir dos resultados encontrados nos ensaios biológicos e análises químicas de 16 alimentos (semente de girassol, aveia, gema de ovo, ovo integral, clara de ovo, germe de trigo, farelo de trigo, milho moído, milho gelatinizado, farelo de girassol, levedura, polpa cítrica, mamão, banana, farelo de soja e soja micronizada), equações para a predição da energia metabolizável aparente (EMA), energia metabolizável corrigida para nitrogênio (EMAn), energia metabolizável verdadeira (EMV) e energia metabolizável verdadeira corrigida para nitrogênio (EMVn) para papagaios verdadeiros (Amazona aestiva) adultos, em manutenção. Baseando-se nas análises bromatológicas de 16 alimentos e nos valores de energia metabolizável (EMA, EMV, EMAn e EMVn) obtidos em papagaios, foram desenvolvidas equações de predição para energia metabolizável de alimentos comumente utilizados em rações e dietas de psitacídeos. Os parâmetros utilizados para os cálculos das equações foram os valores de Matéria Orgânica (MO), Proteína Bruta (PB), Energia Bruta (EB), Fibra Bruta (FB), Extrato Etéreo (EE), Cinzas ou minerais totais $(\mathrm{Cz})$ e Extrativos Não Nitrogenados (ENN), analisados nos alimentos. As equações para predizer a energia metabolizável dos grupos de alimentos foram estimadas através de regressões lineares simples e múltiplas, utilizando-se método de stepwise do pacote estatístico SAS Institute (1995). Os resultados obtidos permitiram concluir que: 1- o EE foi a variável que melhor se correlacionou nas estimativas da energia metabolizável (EMA, EMAn, EMV e EMVn) com alta correlação positiva; 2- embora várias equações tenham apresentado $\mathrm{R}^{2}$ acima de 0,90 , nenhuma delas foi hábil em predizer a energia metabolizável de todos os alimentos avaliados, provavelmente pela natureza heterogênea dos princípios nutritivos dos alimentos.
\end{abstract}

Termos para indexação: Equações, energia metabolizável, psitacídeos.

\section{ABSTRACT}

The objective of this study was to predict the energetics values by using the data set from the metabolism assay and proximal analyses of 16 feeds (sunflower seed, oat, egg yolk, integral egg, egg white, wheat germen, wheat bran, triturated corn, jellied corn, sunflower bran, yeast, citric pulp, papaya, banana, soybean meal, extruded soybean). The prediction equations of the apparent metabolizable energy (AME), apparent nitrogen corrected (AMEn), true (TME) and true nitrogen corrected (TMEn) for blue fronted parrots (Amazon aestiva) adults in the maintenance. Based on the chemical analyses of 16 feeds and in the of metabolizable energy values (AME, TME, AMEn and TMEn) obtained from the assays with parrots, the prediction equations were determined. The parameters used for the calculations of the equations were Organic Matter (OM), Crude Protein (CP), Crude Energy (CE), Crude Fiber (CF), Ethereal Extract (EE), Ashes (As) and Free Nitrogen Extractive (FNE), analyzed in the feds. The equations to predict the metabolizable energy of the groups of feeds were determined through simple and multiple linear regressions, by using the method of stepwise of the SAS statistical package (SAS INSTITUTE, 1995). Accord to the equation it was concluded that : 1 - EE was the variable that was better correlated with the metabolizable energy (AME, AMEn, TME and TMEn) with high positive correlation; 2 - although many equations showed the $\mathrm{R}^{2}$ above 0,90 , none of them was able to predict the metabolizable energy for all feeds evaluated, probably due to the heterogeneous nature of the nutrients in the feeds.

Index terms: Equation, psittacines, metabolizable energy.

(Recebido em 4 de novembro de 2005 e aprovado em 4 de abril de 2006)

\section{INTRODUÇÃO}

A determinação dos valores de energia metabolizável dos alimentos é de suma importância, por ser a forma que representa, da maneira mais adequada, a quantidade de energia disponível nos alimentos para aves. A precisão destes valores está diretamente relacionada com o método de determinação dos valores energéticos e valores precisos são imprescindíveis na elaboração das dietas (ALBINO, 1991, citado por RODRIGUES et al., 2002).

\footnotetext{
Zootecnista, D.Sc.Nutrição de Animais Silvestres - AnimalNutri (Consultoria em Nutrição Animal) - Rua Dr. Armando Amaral, 122 - Bairro Padre Dehon - 37.200-000 - Lavras, MG - eduardosaad@animalnutri.com.br; saadzoo@ufla.br

${ }^{2}$ Zootecnista, D. Sc., Professor Adjunto, Escola de Veterinária da Universidade Federal de Minas Gerais/UFMG - Av. Antônio Carlos, 6627 - Bairro Pampulha - 30.123-970 - Belo Horizonte - waltermf@vet.ufmg.br

${ }^{3}$ Médica Veterinária, D.Sc., Professora Adjunta, Departamento de Zootecnia, da Universidade Federal de Lavras/UFLA - Cx. P. 3037 - $37200-000$ Lavras, MG - borgesvet@ufla.br

${ }^{4}$ Médico Veterinário, D.Sc., Nutriara - Rua Jurutau, 1800 - Bairro Parque Industrial II - Arapongas, PR - leoboscoli@gmail.com
} 
Formulações de rações que utilizam valores imprecisos refletem diretamente no desenvolvimento e manutenção das aves.

Segundo Rodrigues et al. (2002), para aves domésticas, há vários anos, a possibilidade de se utilizar equações para predizer os valores energéticos dos alimentos tem sido alvo de pesquisas. Vários pesquisadores têm desenvolvido equações para estimar a energia metabolizável por meio de sua composição proximal (NRC, 1994).

Sakamura \& Silva (1998) citam que os conteúdos em nutrientes de vários cereais encontrados nas tabelas de composição de alimentos não são confiáveis para a formulação de rações para aves domésticas e, entre os fatores que determinam essa diversidade de valores, podese citar a variedade existente de cultivares.

Para animais silvestres, poucos dados de literatura sobre o valor energético dos alimentos são encontrados e as equações de predição podem ser uma boa ferramenta para o estabelecimento do valor nutritivo dos alimentos para esses animais. Segundo a AAFCO (1998), os valores nutricionais de alimentos para psitacídeos foram estabelecidos, em sua grande maioria, em experimentos com aves domésticas. Essa prática pode estabelecer parâmetros não confiáveis para escolha dos ingredientes na elaboração da dieta para essas aves.

Segundo Borges et al. (2003), mesmo quando se trata de formulações para aves domésticas, torna-se inseguro para a indústria utilizar os valores de tabela e seria extremamente oneroso e difícil submeter todas as partidas de matéria-prima a ensaios "in vivo". Uma vez que estas mesmas indústrias podem obter, com relativa facilidade, determinações químicas como teor de proteína bruta (PB), fibra bruta (FB), extrato etéreo (EE), etc., a utilização de regressões baseadas nessas análises poderia ser de grande valia.

As variáveis químicas mais comumente utilizadas para a estimativa da energia metabolizável dos alimentos são a fibra bruta (FB), fibra detergente ácido (FDA), fibra detergente neutro (FDN), extrato etéreo (EE), proteína bruta $(\mathrm{PB})$, cinzas $(\mathrm{Cz})$ e extrativos não nitrogenados (ENN).

Assim, objetivou-se com esse trabalho determinar equações para a predição da energia metabolizável (EMA, EMAn, EMV e EMVn) dos alimentos e matérias-primas utilizadas nas formulações de rações para papagaios-verdadeiros, a partir dos resultados encontrados nos ensaios biológicos e análise química dos alimentos.

\section{MATERIAL E MÉTODOS}

Baseando-se nas análises químicas dos alimentos (CUNNIFF, 1995) e nos valores de energia metabolizável (EMA, EMV, EMAn e EMVn) obtidos por Saad (2003) no experimento II, que foi conduzido nas dependências do departamento de Zootecnia da Escola de Veterinária da Universidade Federal de Minas Gerais (UFMG), Campus da Pampulha, em Belo Horizonte, em três períodos, em que foram utilizados 34 papagaios-verdadeiros (Amazona aestiva) sexados, distribuídos em blocos ao acaso, em 17 tratamentos, três períodos experimentais, totalizando seis repetições por tratamento (102 unidades experimentais). Foram desenvolvidas equações de predição para energia metabolizável de alimentos comumente utilizados em rações e dietas de psitacídeos. Os parâmetros utilizados para os cálculos das equações foram os valores de Matéria Orgânica (MO), Proteína Bruta (PB), Energia Bruta (EB), Fibra Bruta (FB), Extrato Etéreo (EE), Cinzas ou minerais totais $(\mathrm{Cz})$ e Extrativo Não Nitrogenado (ENN), obtidos por meio da análise de 16 alimentos (Tabela 1).

As equações de predição da energia metabolizável dos alimentos foram estimadas por meio de regressões lineares simples e múltiplas, utilizando-se as análises químicas e os dados de EM dos alimentos pelo método de stepwise do pacote estatístico SAS Institute (1995), método este que fornece a contribuição de cada variável dentro da análise de regressão múltipla. O método mostra a equação que melhor representa a variável dependente estudada e, pela significância do teste $\mathrm{F}$, exclui a variável que menos está contribuindo na determinação do valor energético, até que se obtenha uma equação com apenas uma variável.

Para obter equações de maior precisão, foi adotada uma significância de $15 \%$ de probabilidade para cada variável componente do modelo (valor determinado pelo método de stepwise). Somente foram consideradas as equações em que todas as variáveis independentes apresentassem significância no modelo.

As equações foram obtidas considerando os seguintes agrupamentos: 1 - Valores de EM dos alimentos avaliados, considerando como parâmetros químicos a fibra bruta, proteína bruta, cinzas, extrato etéreo e extrativo não nitrogenado; 2 - Valores de EM dos alimentos avaliados, considerando como parâmetros químicos a matéria orgânica e a energia bruta. 
TABELA 1 - Energia metabolizável aparente (EMA), aparente corrigida pelo nitrogênio (EMAn), verdadeira (EMV) verdadeira corrigida pelo nitrogênio(EMVn), em Kcal $/ \mathrm{kg}$ de MS, observada nos alimentos avaliados e análises laboratoriais de matéria orgânica (\%), energia bruta (Kcal/kg MS), proteína bruta (\%), fibra bruta (\%), extrato etéreo (\%), cinzas $(\%)$ e extrativo não nitrogenado $(\%) .{ }^{1,2}$

\begin{tabular}{|c|c|c|c|c|c|c|c|c|c|c|c|}
\hline Alimentos & EMA & EMAn & EMV & EMVn & EB & MO & PB & FB & $\mathbf{E E}$ & $\mathbf{C z}$ & ENN \\
\hline Semente de Girassol ${ }^{4}$ & 7049,6 & 6884,1 & 7189,7 & 6942,0 & 7643 & 93,04 & 24,17 & 2,86 & 57,80 & 2,98 & 12,19 \\
\hline Aveia & 3846,4 & 3765,9 & 4006,5 & 3886,6 & 4873 & 86,96 & 13,95 & 1,68 & 9,12 & 2,29 & 72,96 \\
\hline Gema de ovo & 5469,2 & 5298,6 & 5722,0 & 5452,9 & 6469 & 91,25 & 38,69 & ND & 46,87 & 6,14 & 8,31 \\
\hline Ovo Integral & 5040,0 & 4781,1 & 5186,0 & 4892,1 & 6228 & 91,25 & 51,84 & ND & 35,41 & 4,60 & 8,16 \\
\hline Clara de ovo & 3433,1 & 3124,3 & 3518,9 & 3211,6 & 5081 & 86,36 & 88,72 & ND & 0,66 & 7,96 & 2,66 \\
\hline Germe de trigo & 3043,8 & 2930,2 & 3106,7 & 3011,0 & 4923 & 83,87 & 29,46 & 2,42 & 9,18 & 5,39 & 53,56 \\
\hline Farelo de trigo & 2021,1 & 2025,4 & 2036,7 & 2086,5 & 4781 & 84,08 & 15,69 & 9,35 & 5,17 & 5,44 & 64,36 \\
\hline Milho moído & 3634,8 & 3647,4 & 3800,3 & 3771,3 & 4495 & 86,47 & 9,01 & 1,19 & 5,07 & 1,14 & 83,60 \\
\hline Milho gelatinizado & 4059,7 & 3998,5 & 4147,5 & 4088,5 & 4512 & 89,24 & 8,84 & 1,46 & 2,38 & 1,31 & 86,01 \\
\hline Farelo de girassol & 1900,0 & 1773,2 & 1922,5 & 1835,9 & 4743 & 87,13 & 27,24 & 27,35 & 3,44 & 4,25 & 37,72 \\
\hline Levedura de Cerveja & 2893,8 & 2700,9 & 2945,4 & 2776,1 & 4688 & 83,55 & 44,82 & ND & 0,68 & 7,30 & 47,20 \\
\hline Polpa cítrica & 1025,5 & 1069,5 & 1059,5 & 1138,3 & 4389 & 84,29 & 11,66 & 9,43 & 3,36 & 5,09 & 70,46 \\
\hline Mamão Desidratado & 3306,2 & 3286,1 & 3414,9 & 3382,5 & 4150 & 92,36 & 1,95 & ND & 1,03 & 1,99 & 95,04 \\
\hline Banana Desidratada & 3284,2 & 3297,9 & 3420,7 & 3407,3 & 4163 & 87,48 & 5,45 & ND & 0,32 & 4,63 & 89,60 \\
\hline Farelo de soja & 2792,2 & 2634,2 & 2921,6 & 2742,9 & 4820 & 82,68 & 50,41 & 4,81 & 3,85 & 5,94 & 34,99 \\
\hline Soja micronizada & 4272,8 & 4081,4 & 4394,2 & 4182,8 & 5815 & 90,56 & 41,92 & 0,49 & 25,19 & 4,56 & 27,85 \\
\hline
\end{tabular}

1-Dados expressos na base da matéria seca.

2-Análises realizadas no Laboratório de Nutrição da Escola de Veterinária da UFMG e no Laboratório de Nutrição do Instituto Mineiro de Agropecuária.

3-MS=matéria seca, $\mathrm{MO}=$ matéria orgânica, $\mathrm{EB}=$ =nergia bruta, $\mathrm{PB}=$ proteína bruta, $\mathrm{FB}=$ fibra bruta, $\mathrm{EE}=\mathrm{extrato}$ etéreo, $\mathrm{Cz}=\mathrm{cinzas}$, ENN=Extrativo não nitrogenado, $\mathrm{Ca}=$ Cálcio e $\mathrm{P}=$ Fósforo.

4-Valores na semente de girassol descorticada manualmente.

\section{RESULTADOS E DISCUSSÃO}

Na Tabela 2, encontram-se as equações de predição para estimativa de energia metabolizável aparente (EMA) a partir de análises químicas (EE, FB, Cz, ENN e PB) dos 16 alimentos avaliados.

As equações com maior número de variáveis no modelo apresentaram coeficientes de determinação $\left(\mathrm{R}^{2}\right)$ mais elevados, sendo 0,907 para as equações com quatro variáveis (EE, FB, Cz e ENN), comparados a 0,865 (EE, FB e $\mathrm{Cz}$ ), 0,82 (EE e FB) e 0,71 (EE) nas equações com três, duas e uma variável, respectivamente. Somente foram consideradas as equações em que todos os componentes do modelo apresentassem significância a $15 \%$ de probabilidade no teste F. O extrato etéreo foi a variável com maior significância no modelo, $(\mathrm{P}=0,0001)$, conforme demonstrado na Tabela 2 , porém a equação resultante $(\mathrm{EMA}=2684,32+67,4 \mathrm{EE})$ apresentou um coeficiente de determinação $\left(\mathrm{R}^{2}\right)$ mediano $(0,71)$ quando comparado às equações com maior número de variáveis. Estes resultados foram semelhantes aos de Campbell et al. (1986), que ao utilizarem oitenta e seis dietas avícolas para desenvolver equações de predição para a EMV, EMVn e EMAn, também encontraram que o EE foi a variável que mais contribuiu no modelo.

Se, neste experimento a melhor variável, com maior contribuição na estimativa de EMA, foi o EE, no trabalho de Borges et al. (2003), com frangos de corte, ao contrário, a FB foi a variável que melhor explicou os valores de EMA, embora sozinha não tenha sido uma boa variável para predizê-la, com um $\mathrm{R}^{2}$ de 0,59 quando a equação foi obtida a partir de dados gerados por todos os métodos de avaliação (colheita total de excreta e alimentação forçada).

A única variável testada que não apresentou significância em uma probabilidade de $15 \%$ foi a proteína 
bruta, descartada pelo método de stepwise para o modelo.

Além das variáveis químicas (PB, FB, EE, Cz e ENN), a energia bruta (EB) e a MO foram utilizadas para calcular equações de predição dos 16 alimentos avaliados (Tabela 3). A equação obtida com EB dos alimentos para EMA foi de $-2869,74+1,26$ EB com $R^{2}$ de 0,68 , mostrandose inferior a qualquer uma das equações obtidas quando se utilizaram as análises químicas, enquanto que a incorporação da matéria orgânica ao modelo aumentou o $\mathrm{R}^{2}$ para 0,80 .

Face às dificuldades de obtenção da EB e às facilidades na obtenção de valores como os de fibra bruta e extrato etéreo e, ainda, aos $\mathrm{R}^{2}$ semelhantes, não se justificam as recomendações para utilização de EB como valor para estimativa de EMA. Também porque as variáveis EE e FB explicaram mais de $80 \%$ da variação nos valores de EMA, reforçando sua utilização para estimativa da EMA.

Da mesma forma que para EMA, o EE foi a variável que mais contribuiu no modelo de estimativa de EMAn (Tabela 4) com um $\mathrm{R}^{2}$ de 0,71 . Quando se somou a FB à equação, estas duas variáveis justificaram 82,15\% das variações. Entretanto, nenhuma outra variável $(\mathrm{Cz}, \mathrm{PB}$ e ENN) foi significante a $\mathrm{P}<0,15$. Rodrigues et al. (2002), estimando equações para soja e seus subprodutos, encontraram que a equação composta por duas variáveis, EE e FB, explicou $92,7 \%$ das variações, mostrando que o ajuste de um modelo com duas variáveis pode ser bem aplicado na estimativa da energia dos alimentos.

TABELA 2 - Regressões para estimativa de energia metabolizável aparente (EMA) a partir de análises químicas (EE, $\mathrm{FB}, \mathrm{Cz}, \mathrm{ENN}$ e PB) $)^{1}$ dos 16 alimentos avaliados.

\begin{tabular}{lcccccc}
\hline & $\mathbf{X 1}$ & $\mathbf{X 1}+\mathbf{X 2}$ & $\mathbf{X 1}+\mathbf{X} 2+\mathbf{X 3}$ & $\mathbf{X 1}+\mathbf{X 2}+\mathbf{X 3}+\mathbf{X 4}$ & $\mathbf{R}^{\mathbf{2}}$ da variável & P>F \\
\hline Intercepta (a) & 2684,32 & 3037,73 & 3688,33 & 5942,39 & - & - \\
EE (X1) & 67,40 & 61,75 & 61,30 & 36,64 & 0,7055 & 0,0001 \\
FB (X2) & - & $-73,23$ & $-72,72$ & $-85,67$ & 0,1188 & 0,0110 \\
Cz (X3) & - & - & $-145,71$ & $-339,82$ & 0,0410 & 0,0802 \\
ENN (X4) & - & - & - & $-20,54$ & 0,0425 & 0,0458 \\
\hline $\mathrm{R}^{2}$ da regressão & 0,7055 & 0,8243 & 0,8653 & 0,9078 & - & - \\
\hline $\mathrm{P}>\mathrm{F}^{3}$ & 0,0001 & 0,0001 & 0,0001 & 0,0001 & - & - \\
\hline
\end{tabular}

Em que $\mathrm{Y}=$ EMA do alimento.

1-PB-Proteína bruta, FB-Fibra Bruta, Cz-Cinzas, EE-Extrato Etéreo e ENN-Extrativo não nitrogenado.

2-Significância (Prob $>F)$ das variáveis e 4-significância (Prob $>F)$ da regressão.

3-A proteína bruta não apresentou significância de $\mathrm{P}<0,15$ e não entrou no modelo.

TABELA 3 - Regressões para estimativa de energia metabolizável aparente (EMA) a partir de análises químicas (MO e EB) ${ }^{1}$ dos 16 alimentos avaliados.

\begin{tabular}{lcccc}
\hline & $\mathbf{X 1}$ & $\mathbf{X 1}+\mathbf{X} 2$ & $\mathbf{R}^{\mathbf{2}}$ da variável & $\mathbf{P}_{\mathbf{2}} \mathbf{F}^{\mathbf{2}}$ \\
\hline Intercepta (a) & $-2869,74$ & $-16887,24$ & - & - \\
EB (X1) & 1,26 & 0,889 & 0,6811 & 0,0001 \\
MO (X2) & - & 181,78 & 0,1190 & 0,0156 \\
\hline $\mathrm{R}^{2}$ da regressão & 0,6811 & 0,8000 & - & - \\
\hline $\mathrm{P}>\mathrm{F}^{3}$ & 0,0001 & 0,0001 & - & - \\
\hline
\end{tabular}

Em que $\mathrm{Y}=$ EMA do alimento.

$1-\mathrm{MO}=$ matéria orgânica e $\mathrm{EB}=$ energia bruta.

2-Significância (Prob>F) das variáveis e 3-significância (Prob>F) da regressão. 
As equações obtidas a partir da EB e MO, considerando os valores de EMAn, encontram-se na Tabela 5. Semelhante ao encontrado para EMA, a equação somente com EB a partir dos valores de EMAn apresentou um $R^{2}$ de 0,71 , enquanto que a incorporação da matéria orgânica ao modelo aumentou o $\mathrm{R}^{2}$ para 0,80 .

Já para a EMV, as equações estimadas com as variáveis EE, $\mathrm{FB}, \mathrm{PB}, \mathrm{CZ}$ e ENN, encontram-se na Tabela 6.

Novamente o EE foi, isoladamente, a variável com maior significância no modelo $(\mathrm{EMV}=2766,09+69,4 \mathrm{EE}$ com um $\mathrm{R}^{2}$ de 0,70 ). Quando outras variáveis foram incorporadas ao modelo, o coeficiente de determinação mostrou-se maior: 0,83 para $\mathrm{EE}+\mathrm{FB}, 0,87$ para $\mathrm{EE}, \mathrm{FB}$ e $\mathrm{Cz}$ e 0,91 para equações com quatro variáveis (EE, $\mathrm{FB}, \mathrm{Cz}$ e ENN). A PB não apresentou significância ao nível de $\mathrm{P}<$ 0,15 sendo descartada do modelo.

Quando se utilizou a EB e MO para estimar a EMV (Tabela 7), repetiu-se o encontrado nas equações para estimativa de EMA e EMAn, com $\mathrm{R}^{2}$ medianos quando se utilizou o valor de todos os alimentos $(0,67$ e 0,79 para EB e $\mathrm{EB}+\mathrm{MO}$, respectivamente).

Finalmente, as equações para calcular a EMVn encontram-se nas Tabelas 8 (variáveis EE, $\mathrm{FB}, \mathrm{Cz}, \mathrm{ENN}$ e PB) e Tabela 9 (variáveis EB e MO).

TABELA 4 - Regressões para estimativa de energia metabolizável aparente corrigida pelo nitrogênio (EMAn) a partir de análises químicas (EE, FB, Cz, ENN e PB) ${ }^{1}$ dos 16 alimentos avaliados.

\begin{tabular}{lcccc}
\hline & $\mathbf{X 1}$ & $\mathbf{X 1}+\mathbf{X 2}$ & $\mathbf{R}^{\mathbf{2}}$ da variável & $\mathbf{P} \mathbf{F} \mathbf{F}^{\mathbf{2}}$ \\
\hline Intercepta (a) & 2559,82 & 2978,79 & - & - \\
EE $(\mathrm{X} 1)$ & 70,61 & 62,87 & 0,7092 & 0,0001 \\
FB $(\mathrm{X} 2)$ & - & $-76,37$ & 0,1123 & 0,0134 \\
\hline $\mathrm{R}^{2}$ da regressão & 0,7092 & 0,8215 & - & - \\
\hline $\mathrm{P}>\mathrm{F}^{3}$ & 0,0001 & 0,0001 & - & - \\
\hline
\end{tabular}

Em que $\mathrm{Y}=\mathrm{EMAn}$ do alimento.

1-PB-Proteína bruta, FB-Fibra Bruta, Cz-Cinzas, EE-Extrato Etéreo e ENN-Extrativo não nitrogenado.

2-Significância (Prob>F) das variáveis e 3-significância (Prob $>F)$ da regressão.

As outras variáveis não apresentaram significância de $\mathrm{P}<0,15$ e não entraram no modelo.

TABELA 5 - Regressões para estimativa de energia metabolizável aparente corrigida pelo nitrogênio (EMAn) a partir de análises químicas (MO e EB) ${ }^{1}$ dos 16 alimentos avaliados.

\begin{tabular}{lcccc}
\hline & $\mathbf{X 1}$ & $\mathbf{X 1}+\mathbf{X 2}$ & $\mathbf{R}^{\mathbf{2}}$ da variável & $\mathbf{P}>\mathbf{F}^{\mathbf{2}}$ \\
\hline Intercepta (a) & $-3363,61$ & $-16942,27$ & - & - \\
EB (X1) & 1,34 & 0,98 & 0,7062 & 0,0001 \\
MO (X2) & - & 176,09 & 0,1023 & 0,0206 \\
\hline $\mathrm{R}^{2}$ da regressão & 0,7062 & 0,8084 & - & - \\
\hline $\mathrm{P}>\mathrm{F}^{3}$ & 0,0001 & 0,0001 & - & - \\
\hline
\end{tabular}

Em que $\mathrm{Y}=\mathrm{EMAn}$ do alimento.

$1-\mathrm{MO}=$ matéria orgânica e $\mathrm{EB}=$ energia bruta.

2-Significância $($ Prob $<$ F) das variáveis e 3-significância $($ Prob $<$ F) da regressão.

Ciênc. agrotec., Lavras, v. 31, n. 3, p. 876-882, maio/jun., 2007 
TABELA 6 - Regressões para estimativa de energia metabolizável verdadeira (EMV) a partir de análises químicas (EE, $\mathrm{FB}, \mathrm{Cz}, \mathrm{ENN}$ e PB) $)^{1}$ dos 16 alimentos avaliados.

\begin{tabular}{lcccccc}
\hline & $\mathbf{X 1}$ & $\mathbf{X 1}+\mathbf{X 2}$ & $\mathbf{X 1}+\mathbf{X} 2+\mathbf{X 3}$ & $\mathbf{X 1}+\mathbf{X 2}+\mathbf{X 3}+\mathbf{X} 4$ & $\mathbf{R}^{\mathbf{2}}$ da variável & $\mathbf{P}>\mathbf{F}^{\mathbf{2}}$ \\
\hline Intercepta (a) & 2766,09 & 3139,33 & 3816,05 & 6105,97 & - & - \\
EE (X1) & 69,37 & 63,40 & $-151,56$ & 37,89 & 0,7031 & 0,0001 \\
FB (X2) & - & $-77,34$ & $-76,82$ & $-89,97$ & 0,1246 & 0,0090 \\
Cz (X3) & - & - & 62,94 & $-348,76$ & 0,0417 & 0,0738 \\
ENN (X4) & - & - & - & $-20,87$ & 0,0413 & 0,0456 \\
\hline $\mathrm{R}^{2}$ da regressão & 0,7031 & 0,8277 & 0,8694 & 0,9107 & - & - \\
\hline $\mathrm{P}>\mathrm{F}^{3}$ & 0,0001 & 0,0001 & 0,0001 & 0,0001 & - & - \\
\hline
\end{tabular}

Em que $\mathrm{Y}=\mathrm{EMV}$ do alimento.

1-PB- Proteína bruta, FB- Fibra Bruta, Cz- Cinzas, EE- Extrato Etéreo e ENN- Extrativo não nitrogenado.

2-Significância (Prob>F) das variáveis e 3-significância (Prob $>$ F) da regressão.

A proteína bruta não apresentou significância de $\mathrm{P}<0,15$ e não entrou no modelo.

TABELA 7 - Regressões para estimativa de energia metabolizável verdadeira (EMV) a partir de análises químicas (MO e EB $)^{1}$ dos 16 alimentos avaliados.

\begin{tabular}{lcccc}
\hline & $\mathbf{X 1}$ & $\mathbf{X 1}+\mathbf{X 2}$ & $\mathbf{R}^{\mathbf{2}}$ da variável & $\mathbf{P}>\mathbf{F}^{\mathbf{2}}$ \\
\hline Intercepta (a) & $-2914,80$ & $-17500,39$ & - & - \\
EB (X1) & 1,29 & 0,90 & 0,6715 & 0,0001 \\
MO (X2) & - & 189,15 & 0,1212 & 0,0164 \\
\hline $\mathrm{R}^{2}$ da regressão & 0,6715 & 0,7926 & - & - \\
\hline $\mathrm{P}>\mathrm{F}^{3}$ & 0,0001 & 0,0001 & - & - \\
\hline
\end{tabular}

Em que $\mathrm{Y}=\mathrm{EMV}$ do alimento.

$1-\mathrm{MO}=$ matéria orgânica e $\mathrm{EB}=$ energia bruta.

2-Significância (Prob< F) das variáveis e 3-significância (Prob< F) da regressão.

TABELA 8 - Regressões para estimativa de energia metabolizável verdadeira corrigida pelo nitrogênio (EMVn) a partir de análises químicas (EE, FB, Cz, ENN e PB) ${ }^{1}$ dos 16 alimentos avaliados.

\begin{tabular}{lcccc}
\hline & $\mathbf{X 1}$ & $\mathbf{X 1}+\mathbf{X 2}$ & $\mathbf{R}^{\mathbf{2}}$ da variável & $\mathbf{P} \mathbf{2} \mathbf{F}^{\mathbf{2}}$ \\
\hline Intercepta (a) & 2646,51 & 3014,46 & - & - \\
EE (X1) & 72,00 & 66,12 & 0,7010 & 0,0001 \\
FB (X2) & - & $-76,24$ & 0,1121 & 0,0152 \\
\hline $\mathrm{R}^{2}$ da regressão & 0,7010 & 0,8131 & - & - \\
\hline $\mathrm{P}>\mathrm{F}^{3}$ & 0,0001 & 0,0001 & - & - \\
\hline
\end{tabular}

Em que $\mathrm{Y}=\mathrm{EMVn}$ do alimento.

1-PB-Proteína bruta, FB-Fibra Bruta, Cz-Cinzas, EE-Extrato Etéreo e ENN-Extrativo não nitrogenado.

2-Significância (Prob>F) das variáveis e 3-significância (Prob $>F)$ da regressão.

A proteína bruta não apresentou significância de $\mathrm{P}<0,15$ e não entrou no modelo. 
TABELA 9 - Regressões para estimativa de energia metabolizável verdadeira corrigida pelo nitrogênio (EMVn) a partir de análises químicas (MO e EB) ${ }^{1}$ dos 16 alimentos avaliados.

\begin{tabular}{lcccc}
\hline & $\mathbf{X 1}$ & $\mathbf{X 1}+\mathbf{X 2}$ & $\mathbf{R}^{2}$ da variável & $\mathbf{P} \mathbf{F}^{\mathbf{2}}$ \\
\hline Intercepta (a) & $-3480,73$ & $-16674,96$ & - & - \\
EB (X1) & 1,34 & 1,04 & 0,7155 & 0,0001 \\
MO (X2) & - & 169,80 & 0,0904 & 0,0287 \\
\hline $\mathrm{R}^{2}$ da regressão & 0,7155 & 0,8058 & - & - \\
\hline $\mathrm{P}>\mathrm{F}^{3}$ & 0,0001 & 0,0001 & - & - \\
\hline
\end{tabular}

Em que $\mathrm{Y}=\mathrm{EMVn}$ do alimento.

$1-\mathrm{MO}=$ matéria orgânica e EB = energia bruta.

2-Significância (Prob>f) das variáveis e 3-significância (Prob>F) da regressão.

\section{CONCLUSÕES}

Os resultados obtidos neste estudo permitem as seguintes conclusões:

O EE foi a variável que melhor se correlacionou nas estimativas da energia metabolizável (EMA, EMAn, EMV e EMVn) com alta correlação positiva (mínimo de 68 e máximo de $91 \%$ ), participando de todos modelos, sendo recomendável sua inclusão em equações de predição energética de alimentos usuais na alimentação de papagaios.

Embora várias equações tenham apresentado $\mathrm{R}^{2}$ acima de 0,90 , nenhuma delas foi hábil em predizer a energia metabolizável de todos os alimentos avaliados, provavelmente pela natureza heterogênea dos princípios nutritivos dos alimentos.

Para pesquisas futuras sugere-se que as equações sejam obtidas utilizando-se alimentos de grupos semelhantes, como subprodutos de uma mesma origem.

\section{REFERÊNCIAS BIBLIOGRÁFICAS}

\section{ASSOCIATION OF AMERICAN FEED CONTROL} OFFICIALS INCORPORATED. Nutrition expert panel review: new rules for feeding pet birds. Official Publication - Feed Management, Atlanta, v. 49, n. 2, 1998.

BORGES, F. M. O.; ROSTAGNO, H. S.; RODRIGUEZ, N. M.; SAAD, C. E. P.; TEIXEIRA, E. A.; ARAUJO, V. L. Valores energéticos do grão de trigo e seus subprodutos para frangos de corte. Arquivos Brasileiros de Medicina Veterinária e Zootecnia, Belo Horizonte, v. 55, n. 6, p. 710$721,2003$.
CAMPBELL, G. L.; SALMON, R. E.; CLASSEN, H. L. Prediction of metabolizable energy of broiler diets from chemical analysis. Poultry Science, Champaign, v. 65, n. 11, p. 2126-2134, 1986.

CUNNIFF, P. (Ed.). Official methods of analysis of AOAC International. 16. ed. Arlington: AOAC International, 1995. v. 1.

NATIONAL RESEARCH COUNCIL. Nutrient requirements of poultry. 9. ed. Washington: National Academy, 1994. 155 p.

RODRIGUES, P. B.; ROSTAGNO, H. S.; ALBINO, L. F. T. Valores energéticos da soja e subprodutos da soja, determinados com frangos de corte e galos adultos. Revista Brasileira de Zootecnia, Viçosa, v. 31, n. 4, p. 1771-1782, 2002.

SAS INSTITUTE. SAS user's guide: statistics. Cary, 1995. $956 \mathrm{p}$.

SAAD, C. E. P. Avaliação de alimentos e determinação das necessidades de proteína para manutenção de papagaiosverdadeiros (Amazona aestiva). 2003. 160 f. Tese (Doutorado) - Universidade Federal de Minas Gerais, Belo Horizonte, 2003.

SAKOMURA, N. K.; SILVA, R. Conceitos inovadores aplicáveis à nutrição de não ruminantes. Caderno Técnico da Escola de Veterinária da UFMG, Belo Horizonte, n. 22, p. 125-146, 1998. 